EDITORIAL

\title{
LUTS/BPH and SARS-COV2: when a misunderstanding in the correct physiopathology results in incorrect associations
}

(c) The Author(s), under exclusive licence to Springer Nature Limited 2021

Prostate Cancer and Prostatic Diseases (2022) 25:5-6; https://doi.org/10.1038/s41391-021-00419-z

Lower urinary tract symptoms (LUTS) in male have traditionally been related to bladder outlet obstruction (BOO), most frequently when histological benign prostatic hyperplasia $(\mathrm{BPH})$ progresses through benign prostatic enlargement (BPE) to benign prostatic obstruction (BPO) [1]. The use of a correct terminology is essential to evaluate the pathophysiology of lower urinary tract symptoms and $\mathrm{BPH}$. The pathogenesis and progression of BPH is still not fully understood but it is most likely multifactorial. Increased sympathetic nervous activity, hormonal alterations, the presence of the metabolic syndrome (MetS) and prostatic inflammation are major actors in its development [2,3]. Historically, different viruses and bacteria have been identified in the prostate, and most of them could be responsible for the inflammatory response. Autoimmune diseases, metabolic factors including metabolic syndrome have been also considered as possible initial triggers of the inflammatory response which is observed in the prostate in patients with $\mathrm{BPH}$. So far, theoretically the possibility that SARS-COV2 may be involved in the development of inflammatory infiltrates inside the prostate seems reasonable.

In this issue of Prostate Cancer and Prostatic diseases, Haghpanah et al. presented their systematic review on the possible role of SARSCOV2 in progression of benign prostatic hyperplasia [4]. The search conducted by the authors identified papers highlighting a possible correlation between BPH and SARS-COV2 infection. According to the authors, SARS-COV2 could act on the prostate by activating inflammatory pathways or by the androgen receptor. The authors conclude based on their analysis that SARS-COV2 could act as a promoter of BPH progression. Moreover, they suggest monitoring the progression of symptoms of patients with BPH and LUTS and testing PSA levels in SARS-COV2 patients during the pandemic.

Unfortunately, this conclusion which seems scientifically sound should be mitigated and is to be considered somehow dangerous particularly for the controversial role of PSA testing in the general population. The evidence from the present review is poor and partially reflects of the lack of in vitro or in vivo studies to support their conclusions. All the possible pathways between SARS-COV2 infections and BPH simply reflect previous well-established pathways on the role of viral infections, metabolic syndrome and $\mathrm{BPH}$. Based on this evidence what applies to SARS-COV2 is not specific but applies to several different pathogens agents [5].

The possible reported association between SARS-COV2 infection and metabolic factors seems too simplicistic. Metabolic syndrome (MetS) represents a cluster of metabolic abnormalities related to central adiposity and insulin resistance and it is associated to an increased risks of cardiovascular diseases [6]. Although the exact biological pathway is not clear yet, insulin resistance, increased visceral adiposity, sex hormone alterations and cellular inflammatory reactions play significant roles in the association between metabolic syndrome and prostatic diseases [7]. The proposed pathway where SARS-COV2 infection leads to metabolic abnormalities and subsequent inflammatory infiltrates and BPH presents a population bias. Most of the reported cases of SARS-COV 2 comes from hospitalized patients which are normally older patients with several comorbidities including metabolic abnormalities. The possible effect of metabolic factors as diabetes or obesity on prostatic inflammatory infiltrates also needs several years and is also influenced by diet, lifestyle and concomitant medications. So far, the direct effect on SARS-COV 2 on BPH pathogenesis by inducing metabolic abnormalities is at least questionable.

Furthermore, the relationship between SARS-COV2 and AR is still to be defined. In the recent ASCO-GU congress several presentations evaluating more 7000 patients have clearly demonstrated that androgen levels have no relationship with the risk of SARS-COV2 infection and do not protect against severe SARSCOV2 illness. Standing to these evidences, the relationship between SARS-COV2 and AR is still controversial or minimal. So far, the academic question on the relationship between SARS-COV 2 infection and BPH development and progression is still there. Further in vitro and animal studies should be used to investigate the effect of SARS-COV2 on the prostate as well as longitudinal study which follow-up young - patients after SARS-COV2 infection are needed to estimate the possible effect on this infection on BPH development, LUTS and eventually prostate cancer.

The possible relationship between LUTS and SARS-COV2 infection is even more complicated. LUTS have a multifactorial origin and both occur in men and women with similar prevalence [8]. The only available evidence on this relationship is poor. The authors evaluated a small study by Kaya and colleagues which recorded a statistically significant difference of less than 1 point in IPSS storage score in patients after SARS COV2 infection while no difference in voiding or total IPSS. It is important to remember that statistically significant does not mean clinically significant, in fact a change of less than 3 points is not to be considered clinically relevant [9].

American and European guidelines have clearly stated the diagnostic pathway to be followed in patients with LUTS including the pros and cons of a PSA screening. Furthermore, generally speaking, the drivers for a urological consultation in patients with LUTS/BPH are complex and include several factors as partners, fear of cancer or fear of surgery [10]. At this stage what we known is only the tip of a very large iceberg and based on our current knowledge we cannot support the idea of changing our practice on LUTS/BPH management in relation to the SARS-COV2 infections. SARS-COV2 have completely changed our life, has a significant impact on patients care particularly in uro-oncology but 
at this time has no known impact on LUTS and BPH development or progression. Further evidence and stronger data are needed before any indication on changing our clinical practice in a chronic benign condition such as BPH. As already underlined previously, our effort as clinicians should be focused on diet, exercise and lifestyle in order to impact on cardiovascular disorders and metabolic factors. As a matter of fact, this should have a dramatic impact on cardiovascular morbidity and possibly on prostatic diseases development and progression and last but not least on severe SARS-COV2 incidence. Researchers should avoid sailing in the fogs searching for unusual associations, many safe harbors where to dock already exist in the field of LUTS/BPH but we just need explore them.

$$
\begin{array}{r}
\text { Cosimo De Nunzio } \mathbb{D}^{1 凶}, \text { Antonio Franco }{ }^{1} \text { and } \\
\text { Riccardo Lombardo }
\end{array}
$$

\section{REFERENCES}

1. Abrams P, Cardozo L, Fall M, Griffiths D, Rosier P, Ulmsten U, et al. The standardisation of terminology of lower urinary tract function: report from the Standardisation Sub-committee of the International Continence Society. Neurourol Urodyn. 2002;21:167-78.

2. Gacci M, Sebastianelli A, Salvi M, De Nunzio C, Tubaro A, Vignozzi L, et al. Central obesity is predictive of persistent storage lower urinary tract symptoms (LUTS) after surgery for benign prostatic enlargement: Results of a multicentre prospective study. BJU Int. 2015;116:271-7.

3. De Nunzio C, Cindolo L, Gacci M, Pellegrini F, Carini M, Lombardo R, et al. Metabolic syndrome and lower urinary tract symptoms in patients with benign prostatic enlargement: A possible link to storage symptoms. Urology. 2014;84:1181-7.

4. Fatemeh M, Haghpanah A, Salehipour M, Hosseinpour A, Roozbeh J, Dehghani A. Is COVID-19 a risk factor for progression of benign prostatic hyperplasia and exacerbation of its-related symptoms?: a systematic review. Prostate Cancer Prostatic Dis. 2021; In Press.
5. Simonato A, Giannarini G, Abrate A, Bartoletti R, Crestani A, De Nunzio C, et al. Pathways for urology patients during the COVID-19 pandemic. Minerva Urol Nefrol. 2020. https://doi.org/10.23736/S0393-2249.20.03861-8.

6. Gacci M, Corona G, Sebastianelli A, Serni S, De Nunzio C, Maggi M, et al. Male lower urinary tract symptoms and cardiovascular events: a systematic review and meta-analysis. Eur Urol. 2016;70:788-96.

7. De Monte C, Carradori S, Granese A, Di Pierro GB, Leonardo C, De Nunzio C. Modern extraction techniques and their impact on the pharmacological profile of Serenoa repens extracts for the treatment of lower urinary tract symptoms. BMC Urol. 2014. https://doi.org/10.1186/1471-2490-14-63.

8. Coyne KS, Sexton CC, Thompson CL, Milsom I, Irwin D, Kopp ZS, et al. The prevalence of lower urinary tract symptoms (LUTS) in the USA, the UK and Sweden: results from the Epidemiology of LUTS (EpiLUTS) study. BJU Int. 2009;104:352-60.

9. Kaya Y, Kaya C, Kartal T, Tahta T, Tokgöz VY. Could LUTS be early symptoms of COVID-19. Int J Clin Pract. 2021;75. https://doi.org/10.1111/ijcp.13850.

10. De Nunzio $C$, Presicce $F$, Lombardo $R$, Trucchi $A$, Bellangino $M$, Tubaro $A$, et al. Patient centred care for the medical treatment of lower urinary tract symptoms in patients with benign prostatic obstruction: a key point to improve patients' care-A systematic review. BMC Urol. 2018;18:1-12.

\section{COMPETING INTERESTS}

The authors declare no competing interests.

\section{ADDITIONAL INFORMATION}

Correspondence and requests for materials should be addressed to C.D.N.

Reprints and permission information is available at http://www.nature.com/ reprints

Publisher's note Springer Nature remains neutral with regard to jurisdictional claims in published maps and institutional affiliations. 